\title{
Erratum
}

\section{Exact solution of the neutralino mass matrix}

Monoranjan Guchait

Department of Physics, Jadavpur University, Calcutta 700032, India

Z. Phys. C 57 (1993) 157

The correct form of the following equations and functions should read:

Equation (2): $M_{\mathrm{ch}}=\left(\begin{array}{cc}M & \sqrt{2} M_{w} c_{v} \\ \sqrt{2} M_{w} s_{v} & \mu\end{array}\right)$.

On p. 158 [below Eq. (7)],

$$
\begin{aligned}
R= & \left(\frac{A^{2}}{4}-B+Y\right)^{1 / 2} \\
D_{ \pm}= & \left(\frac{3 A^{2}}{4}-R^{2}-2 B\right. \\
& \left. \pm \frac{4 A B-8 C-A^{3}}{4 R}\right)^{1 / 2} \text { if } R \neq 0
\end{aligned}
$$

$D_{ \pm}=\left(\frac{3 A^{2}}{4}-2 B \pm 2\left(Y^{2}-4 D\right)^{1 / 2}\right)^{1 / 2} \quad$ if $R=0$

and

$Z_{ \pm}=\left\{-\frac{v}{2} \pm\left(\frac{v^{2}}{4}+\frac{u^{3}}{27}\right)^{1 / 2}\right\}^{1 / 3}$

In Eq. (9)

$T_{4 i}=\frac{\xi M_{\chi_{i}^{0}}+M_{z}^{2} s_{v}^{2}}{M_{z} c_{v}\left(M_{\chi_{i}^{0}}+\mu \tan \theta_{v}\right)} \frac{1}{X_{i}^{1 / 2}}$

and

$\xi=\frac{\left(N_{11}-M_{\chi_{i}^{0}}\right)\left(N_{22}-M_{\chi_{i}^{0}}\right)-N_{12} N_{21}}{N_{11}-M_{\chi_{i}^{0}}}$. 